\title{
Serum creatinine/cystatin $C$ ratio as a surrogate marker for sarcopenia in patients with gastric cancer
}

Jing Sun ${ }^{1 \dagger}$, Hui Yang ${ }^{1 \dagger}$, Wentao Cai ${ }^{1 \dagger}$, Jingwei Zheng ${ }^{1}$, Ningzhe Shen ${ }^{1}$, Xinxin Yang ${ }^{1}$, Bujian Pan ${ }^{1}$, Weiteng Zhang ${ }^{1 *}$, Xiaodong Chen ${ }^{1,2^{*}}$ and Xian Shen ${ }^{1,2^{*}}$

\begin{abstract}
Background: Sarcopenia is an age-related syndrome that may have negative impact on surgical outcomes and long-term survival of patients with gastric cancer. Serum creatinine/cystatin C ( Cr/CysC) ratio has attracted attention as a surrogate marker for sarcopenia but has not been adequately studied in patients with gastric cancer. The purpose of this study was to investigate the validity of serum $\mathrm{Cr} / \mathrm{Cys} \mathrm{C}$ ratio as a predictor of sarcopenia, evaluate a statistical cut-off value, and assess the relationship between $\mathrm{Cr} / \mathrm{Cys} \mathrm{C}$ ratio and prognosis of patients with gastric cancer.

Methods: We retrospectively studied 327 patients who underwent surgery for gastric cancer from June 2009 to October 2021. The skeletal muscle mass index was calculated using computed tomography (CT). We determined the relevance of serum $\mathrm{Cr} / \mathrm{Cys} \mathrm{C}$ ratio as a surrogate maker for sarcopenia by comparing it with various biomarkers. The Concordance index (C-index) was calculted to measure whether the $\mathrm{Cr} / \mathrm{Cys} \mathrm{C}$ ratio can prognosis of patients with gastric cancer.

Results: Serum Cr/CysC was significantly correlated with with Skeletal Muscle Index (SMI) $(r=0.221, p<0.001)$ and Skeletal Muscle Area (SMA) $(r=0.258, p<0.001)$. The area under the curve for sarcopenia was significantly larger for serum $\mathrm{Cr} /$ Cys C ratio than for other biomarkers (Cr/CysC: 0.644, CysC: $0.535, \mathrm{Cr}$ : 0.556). Patients in the high-Cr/CysC group have longer survival time than that in low-Cr/CysC group, defined by the cutoff value 0.67 . The $\mathrm{C}$-index of both $\mathrm{Cr} / \mathrm{Cys} \mathrm{C}$ ratio and SMI with OS was 0.63 .

Conclusions: Serum $\mathrm{Cr} / \mathrm{Cys} \mathrm{C}$ ratio can be used accurately, inexpensively, and easily to evaluate sarcopenia in male patients with gastric cancer. Our study shows that patients with $\mathrm{Cr} / \mathrm{Cys} \mathrm{C}$ below 0.67 had possibility of sarcopenia and would be poor prognosis.
\end{abstract}

Keywords: Serum Cr/CysC ratio, Sarcopenia, Gastric cancer

\section{Background}

Gastric cancer is one of the most common malignant tumors worldwide. The incidence (5.7\%) and mortality

\footnotetext{
*Correspondence: jyzwt545@126.com; 15167797063@163.com; shenxian@wmu.edu.cn

${ }^{\dagger}$ Jing Sun, Hui Yang and Wentao Cai are co-author

${ }^{1}$ Department of Gastrointestinal Surgery, The Second Affiliated Hospital,

Wenzhou Medical University, Wenzhou, Zhejiang, China

Full list of author information is available at the end of the article
}

(8.2\%) rates were fifth and third, respectively in 2018 [1]. Although development of technology has led to progress in tumor diagnosis, surgical methods, and adjuvant treatment, the survival rate of patients with gastric cancer remains low [2].

Sarcopenia is an age-related syndrome characterized by progressive and extensive loss of skeletal muscle mass and strength [3]; this has a negative impact on surgical outcomes and long-term survival of patients with gastric cancer [4]. 
Studies have shown that weight loss and malnutrition are common problems in patients with gastric cancer $[5,6]$.

In recent years, researchers have proposed a new method to predict muscle mass. Routinely measured serum creatinine $(\mathrm{Cr})$ and serum cystatin $\mathrm{C}(\mathrm{Cys} \mathrm{C})$ were used to assess renal function. Since the concentration of serum creatinine is affected by muscle mass, patients with decreased muscle mass will decrease serum creatinine $[7,8]$; while serum Cys $C$ is not affected by muscle mass $[9,10]$. Based on the characteristics of these two, the method of dividing serum creatinine by serum Cys $\mathrm{C}$ can be used to further predict muscle mass. This method has been confirmed in the research of many diseases [1114]. However, there are no studies to test the effectiveness of $\mathrm{Cr} / \mathrm{Cys} \mathrm{C}$ in patients with gastric cancer. Based on the above reasons, we are thinking about whether we can explore the prediction of $\mathrm{Cr} / \mathrm{Cys} \mathrm{C}$ for muscle mass in gastric cancer patients, and determine the best cut-off value of $\mathrm{Cr} / \mathrm{CysC}$ ratio for predicting sarcopenia and its impact on the survival time of gastric cancer patients.

\section{Materials and methods Patients and study design}

We obtained patient data from the Second Affiliated Hospital and Yuying Children's Hospital of Wenzhou Medical University from June 2009 to May 2021. Inclusion criteria were as follows: (a) pathological diagnosis of gastric adenocarcinoma, (b) underwent radical gastrectomy, (c) underwent computed tomography $(\mathrm{CT})$ imaging within 1 month preoperatively, and measure the concentration of serum creatinine and serum cystatin $C$ whitin 1 week preoperatively. The exclusion criteria were as follows: (a) a diagnosis of metastatic or remnant gastric cancer, (b) received preoperative chemotherapy or radiotherapy, (c) having incomplete or inaccurate medical records, and (d) renal function impairment (an estimated glomerular filtration rate of $<60 \mathrm{~mL} / \mathrm{min} / 1.73 \mathrm{~m}^{2}$ ). After applying these criteria, 327 patients were recruited. In this study, the treatment of gastric cancer was developed according to the 2010 Japanese Gastric Cancer Treatment Guidelines [5]. Each patient provided their written informed consent to participate in this study. The research protocol was approved by the ethics committee. This research was conducted in accordance with the principles of the Declaration of Helsinki.

\section{Data collection}

We collected the following clinical information from each patient: (1) preoperative personal information, including age, sex, height, American Society of Anesthesiologists (ASA) grade, complications, and preoperative serum creatinine and serum cystatin C; (2) postoperative conditions, including tumor location, tumor size, tumor histology type (tumor differentiation divided into high, medium, and low), pTNM staging, and chemotherapy. Tumor staging conforms to the 8th edition of the corresponding American Joint Committee on Cancer guidelines [15]. To reduce bias, all evaluations were performed by two independent, well-trained researchers and all other data were ignored.

\section{Definition of sarcopenia}

Abdominal CT scan is routinely performed before surgery. Skeletal muscle mass was estimated by calculating the area of all skeletal muscles on the cross-section of the third lumbar vertebra (L3) [16] with ImageJ software. Tissues with a specific Hounsfield unit threshold of -29-150 were considered as skeletal muscles and were standardized according to height $\left(\mathrm{m}^{2}\right)$, and the skeletal muscle mass was evaluated by calculating the skeletal muscle mass index (SMI) $\left(\mathrm{cm}^{2} / \mathrm{m}^{2}\right)$ [17].

The criteria for sarcopenia were low skeletal muscle mass, low muscle strength, and/or poor physical function according to the European Working Group on Sarcopenia in Older People (EWGSOP) [18] and the Asian Working Group for Sarcopenia (AWGS) [19]. We used the following cut-off values to define sarcopenia: (1) SMI (L3) $<40.8$ $\mathrm{cm}^{2} / \mathrm{m}^{2}$ for men and $\mathrm{SMI}<34.9 \mathrm{~cm} 2 / \mathrm{m} 2$ for women, considering possible racial differences indicating low muscle mass [20]. (2) Hand grip strength $<28 \mathrm{~kg}$ among men and $<18 \mathrm{~kg}$ among women were regarded as low muscle strength [19]; and (3) $6-\mathrm{m}$ usual gait speed $<1.0 \mathrm{~m} / \mathrm{s}$ was regarded as low physical performance [19].

\section{Follow-up}

All patients were followed up in the outpatient department in the first month after surgery. After that, the patients were followed up by telephone or in an outpatient clinic every 36 months. The overall survival (OS) was defined as the time between surgery and death from any cause or the date of the last follow-up. We choose the patients from June 2009 to October 2017. All patients were followed up for at least 3 years. The last follow-up was in October 2020.

\section{Laboratory measurements}

Serum Cr levels were measured at our hospital laboratory. Serum $\mathrm{Cr}$ levels were measured using an enzymatic method. Serum cystatin $C$ concentrations were determined by using a particle-enhanced immunoturbidimetric assay, and $\mathrm{Cr} / \mathrm{Cys} \mathrm{C}$ ratio was calculated by diving serum $\mathrm{Cr}$ by serum Cys $\mathrm{C}$.

\section{Statistical analysis}

All continuous data in this study were non-normally distributed according to the Kolmogorov-Smirnov test. 
Therefore, continuous data were presented as median and interquartile range (IQR). Kaplan-Meier survival analysis were used to calculate the effects of $\mathrm{Cr} / \mathrm{CysC}$ ratio on survival time. Receiver operating characteristic (ROC) curve analysis was used to evaluate the utility of the $\mathrm{Cr} / \mathrm{CysC}$ ratio for identifying low SMI, based on the area under the ROC curve (AUC) and 95\% confidence interval (CI). The Y ouden index (sensitivity + specificity-1) was calculated to determine the optimal cutoff points for the $\mathrm{Cr} / \mathrm{CysC}$ ratio. The $\mathrm{C}$-index was calculated to measure the impact of $\mathrm{Cr} / \mathrm{CysC}$ ratio and $\mathrm{SMI}$ on prognosis. All $\mathrm{P}$ values were two-sided, and a $\mathrm{P}$ value of $<0.05$ was used to denote statistical significance. All statistical analyses were performed using Statistical Package for the Social Sciences, version 26.0 (IBM Corp., Armonk, NY, USA) and R version 4.1.2 (The R Foundation, Vienna, Austria).

\section{Results}

\section{Patient characteristics}

We included 339 adults to participate in this study from June 2009 to October 2021. Some participants were excluded because of an estimated glomerular filtration rate of $<60 \mathrm{~mL} / \mathrm{min} / 1.73 \mathrm{~m}^{2}(\mathrm{n}=12)$. A total of 327 participants (median age: 64 years old, range 20-89 years old) were considered eligible for the analysis, including 234 men and 93 women, whose characteristics are shown in Table 1 . There were 26 sarcopenia patients $(11.1 \%)$ out of 234 men, and 16 sarcopenia patients (17.2\%) out of 93 women among the patients. We conducted a correlation analysis on the basic characteristics of the sample. As shown in the heat map (Fig. 1), the serum $\mathrm{CysC} / \mathrm{Cr}$ ratio is positively correlated with SMA, SMI, weight, height, and BMI, and negatively correlated with age and sarcopenia, but there is no strong correlation.

Table 1 Clinical characteristics of the male and female groups

\begin{tabular}{lll}
\hline Factors & Male $(\mathbf{n}=\mathbf{2 3 4})$ & Female $(\mathbf{n = 9 3})$ \\
\hline Age (years) & $63.3 \pm 11.2$ & $59.5 \pm 12.8$ \\
Weight $(\mathrm{kg})$ & $61.9 \pm 11.3$ & $55.0 \pm 8.5$ \\
Height $(\mathrm{m})$ & $1.68 \pm 0.1$ & $1.58 \pm 0.1$ \\
BMI $\left(\mathrm{kg} / \mathrm{m}^{2}\right)$ & $21.8 \pm 3.7$ & $22.0 \pm 3.2$ \\
SMA (CT scan, kg) & $139.3 \pm 20.9$ & $102.4 \pm 18.2$ \\
SMI (kg/m $\left.{ }^{2}\right)$ & $48.9 \pm 7.2$ & $40.9 \pm 7.2$ \\
Serum cystatin C (mg/L) & $1.0 \pm 0.3$ & $0.8 \pm 0.2$ \\
Serum creatinine (mg/dL) & $0.8 \pm 0.2$ & $0.6 \pm 0.1$ \\
Serum Cr/Cys C & $0.8 \pm 0.2$ & $0.7 \pm 0.1$ \\
Sarcopenia, n\% & $11.1 \%$ & $17.2 \%$ \\
\hline
\end{tabular}

\section{Correlation between clinical factors for sarcopenia}

We performed Logistic regression analysis to evaluate whether $\mathrm{Cr} / \mathrm{Cys} \mathrm{C}$ is related to sarcopenia. Univariate analysis showed that $\mathrm{Cr} / \mathrm{Cys} \mathrm{C}$, age, lower BMI, preoperative anemia, tumor size, and pTNM stage are predictors of sarcopenia. And $\mathrm{Cr} / \mathrm{Cys} \mathrm{C}(\mathrm{p}=0.01)$ is still an independent predictor of sarcopenia in multivariate analysis (Table 2). In addition, other markers including age $\geq 75$ years, lower BMI have also been shown to be associated with increased risk of sarcopenia (all $\mathrm{p}<0.05$ ).

\section{Association between various biomarkers and sarcopenia}

Serum $\mathrm{Cr} / \mathrm{CysC}$ ratio was positively correlated with SMI $(\mathrm{r}=0.221, \mathrm{p}<0.001)$ and SMA $(\mathrm{r}=0.258, \mathrm{p}<0.001)$. Serum $\mathrm{Cr}$ was positively correlated with SMI $(r=0.201$, $\mathrm{p}=0.002)$ and SMA $(\mathrm{r}=0.249, \mathrm{p}<0.001)$, while serum CysC had no significant correlation with SMI and SMA ( $>0.05)$ (Fig. 2). We calculated the AUC of each biomarker using the ROC curve (Fig. 3), and used the DeLong test to check the effectiveness of each biomarker as a predictor of sarcopenia. We divided the patients into two groups according to whether to measure hand grip strength and 6-m usual gait speed, a standard group that measured muscle mass and a low skeletal muscle mass group that only measured muscle area. Serum $\mathrm{Cr} / \mathrm{CysC}$ ratio, CysC, Cr AUC were 0.664 (95\% CI 0.511-0.816), 0.630 (95\% CI $0.452-0.808$ ), 0.543 (95\% CI 0.367-0.719) in standard group. And Serum $\mathrm{Cr} / \mathrm{Cys} \mathrm{C}$ ratio, $\mathrm{CysC}, \mathrm{Cr}$ AUC were 0.644 (95\% CI 0.532-0.757), 0.535 (95\% CI 0.418-0.652), 0.556 (95\% CI 0.432-0.681) in low skeletal muscle mass group. The AUC of the $\mathrm{Cr} / \mathrm{CysC}$ ratio in two group were both significantly greater than the AUC of all other biomarkers $(\mathrm{p}=0.034$ and $\mathrm{p}=0.016)$.

\section{Cutoff value of serum $\mathrm{Cr} / \mathrm{CysC}$ on sarcopenia}

We calculated the serum $\mathrm{Cr} / \mathrm{Cys} \mathrm{C}$ cutoff to be 0.67 , using Youden-Index that maximizes the value of "sensitivity $\mathrm{p}$ specificity-1". Based on the calculated cut-off value, in the Low skeletal muscle mass group, the sensitivity, specificity, positive predictive value, and negative predictive value were $0.26,0.87,0.25$ and 0.88 , respectively. And the sensitivity, specificity, positive predictive value, and negative predictive value were $0.44,0.84,0.26$ and 0.92 in the standard group (Fig. 4).

\section{Survival outcomes}

We used the calculated optimal cut-off value to draw the $\mathrm{KM}$ curve of serum $\mathrm{Cr} / \mathrm{CysC}$ and survival time after excluding 2 patients who died within 1 month after surgery in order to reduce the impact of postoperative acute complications on survival time. As shown in the Fig. 5, serum $\mathrm{Cr} / \mathrm{CysC}$ is highly correlated with OS. The OS of 


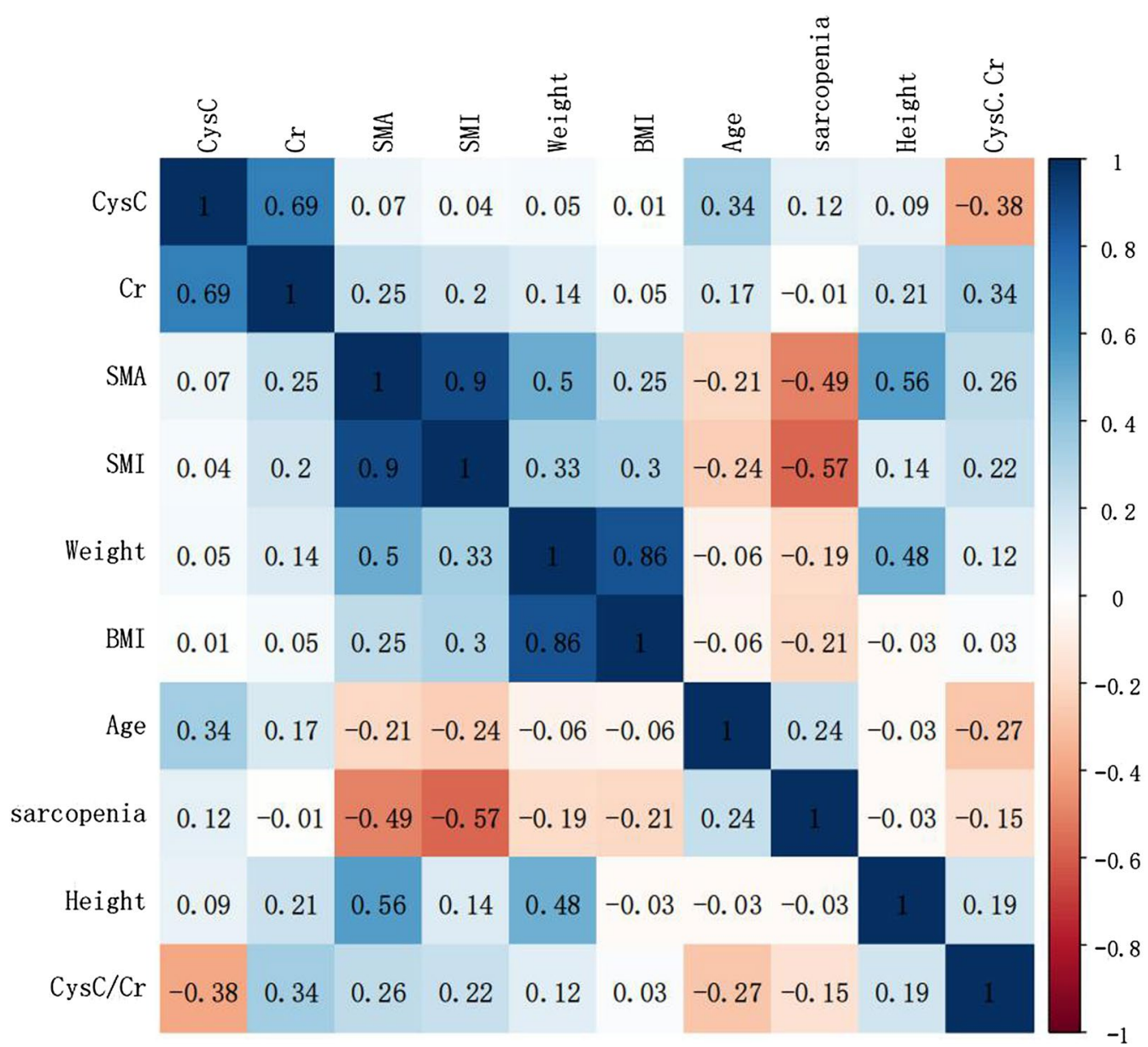

Fig. 1 Correlation heat map analysis between factors which are basic characteristics of the population

the high serum $\mathrm{Cr} / \mathrm{Cys} \mathrm{C}$ group was significantly longer than that of the low serum $\mathrm{Cr} / \mathrm{CysC}$ group $(\mathrm{p}=0.02)$. We used age, BMI, pTNM stage and tumor size to perform COX regression to perform survival multivariate analysis (Table 3). And Cr/Cys C (HR $=0.565$ (95\% CI $0.311-1.025) ; p=0.060)$ is marginal significant, which needs expand the sample size and eliminate confounding factors for further analysis (Table 3). In the $\mathrm{R}$ software, we calculated the height-calibrated $\mathrm{Cr} / \mathrm{CysC}$ ratio, SMI and survival $\mathrm{C}$-index value. The $\mathrm{C}$-index of SMI and OS was 0.62 . And after calibrationed by height, the $\mathrm{C}$-index of $\mathrm{Cr} / \mathrm{Cys} \mathrm{C}$ ratio and $\mathrm{OS}$ was 0.56 . When they both as variables, we calcualted the $\mathrm{C}$-index as 0.63 , which higher than that of SMI.

\section{Discussion}

Baumgartner et al. first proposed the concept of "sarcopenia" [21] to describe the decrease in muscle mass of the elderly with age in 1998. The occurrence of sarcopenia is closely related to age and special physiological conditions which has received extensive attention from scientists in recent years. Many studies have shown that sarcopenia is associated with an increased risk of recurrence [22], shortened survival time [23, 24] and increased other causes of death [25] after gastric cancer resection. Weight loss and malnutrition are issues that are very worthy of our attention in all stages of gastric cancer treatment. More than half of patients with gastric cancer have some degree of weight loss due to the potential impact of the tumor at the time of diagnosis [26]. The inability to maintain weight is considered to be a poor prognostic factor that affects long-term survival during neoadjuvant therapy or chemotherapy [27, 28].

The relationship between $\mathrm{Cr} / \mathrm{CysC}$ ratio and sarcopenia in different populations has been discussed. Tetsuka et al. found for the first time that the $\mathrm{Cr} / \mathrm{Cys} \mathrm{C}$ ratio of patients with amyotrophic lateral sclerosis was lower than that of healthy people [29]. In the Japanese elderly without severe renal impairment, the $\mathrm{Cr} / \mathrm{CysC}$ ratio is positively correlated with muscle mass and physical function [13]. 
Table 2 Univariate and multivariate analyses the risk of sarcopenia

\begin{tabular}{|c|c|c|c|c|}
\hline \multirow[t]{2}{*}{ Factors } & \multicolumn{4}{|c|}{ Odds ratio for sarcopenia $(95 \% \mathrm{Cl})$} \\
\hline & Univariate analysis & p value & Multivariate analysis & $p$ value \\
\hline \multicolumn{5}{|l|}{ Age } \\
\hline$>75 / \leq 75$ & $8.543(4.056-17.998)$ & $<0.001^{*}$ & $10.035(4.217-23.877)$ & $<0.001^{*}$ \\
\hline \multicolumn{5}{|l|}{ Sex } \\
\hline Male/female & $1.662(0.846-3.266)$ & 0.140 & & \\
\hline \multicolumn{5}{|l|}{$\mathrm{BMI}, \mathrm{kg} / \mathrm{m}^{2}$} \\
\hline$\geq 18.5 /<18.5$ & $0.177(0.085-0.369)$ & $<0.001^{*}$ & $0.144(0.062-0.337)$ & $<0.001^{*}$ \\
\hline \multicolumn{5}{|l|}{ Anemia } \\
\hline Yes/no & $2.091(1.032-4.235)$ & $0.041^{*}$ & $1.607(0.705-3.666)$ & 0.259 \\
\hline \multicolumn{5}{|l|}{ Tumor location } \\
\hline Corpus/cardia & $1.012(0.388-2.641)$ & 0.980 & & \\
\hline Pylorus/cardia & $1.020(0.426-2.441)$ & 0.965 & & \\
\hline Mixed or total/cardia & $0.614(0.070-5.415)$ & 0.660 & & \\
\hline \multicolumn{5}{|l|}{ Tumor size } \\
\hline$\geq 4.75 /<4.75$ & $2.100(1.079-4.086)$ & $0.029^{*}$ & $1.975(0.842-4.632)$ & 0.118 \\
\hline \multicolumn{5}{|l|}{ Type of differentiation } \\
\hline Moderate/well & $1.151(0.376-3.528)$ & 0.805 & & \\
\hline Poor/well & $0.955(0.274-3.337)$ & 0.943 & & \\
\hline \multicolumn{5}{|l|}{ pTNM stage } \\
\hline$\| / 1$ & $2.819(1.046-7.595)$ & $0.040^{*}$ & $2.639(0.716-9.731)$ & 0.145 \\
\hline$|1| / \mid$ & $2.409(1.040-5.580)$ & $0.040^{*}$ & $1.905(0.595-6.599)$ & 0.278 \\
\hline \multicolumn{5}{|l|}{ Serum Cr/Cys C } \\
\hline Low/high & $0.336(0.163-0.692)$ & $0.003^{*}$ & $0.334(0.144-0.773)$ & $0.010^{*}$ \\
\hline
\end{tabular}

The reduction of $\mathrm{Cr} / \mathrm{CysC}$ ratio is considered to be a predictor of sarcopenia in patients with type 2 diabetes and COPD [11, 30]. Recent studies have found that the $\mathrm{Cr} /$ $\mathrm{Cys} C$ ratio can also predict malnutrition, weakness and poor clinical outcomes in ICU patients [31-33]. Studies have also confirmed that this indicator can be used as an effective surrogate indicator for evaluating sarcopenia in patients after colorectal cancer surgery [34]. However, there is no report confirming the association between $\mathrm{Cr}$ / $\mathrm{CysC}$ ratio and sarcopenia in patients with gastric cancer.

This study shows that the serum $\mathrm{Cr} / \mathrm{CysC}$ ratio is a useful predictor of sarcopenia compared with other biomarkers (such as serum $\mathrm{Cr}$, CysC). In addition, the best cut-off value of serum $\mathrm{Cr} / \mathrm{CysC}$ ratio is 0.67 . Patients with $\mathrm{Cr} / \mathrm{CysC} \geq 0.67$ can basically rule out sarcopenia.

We found that the $\mathrm{Cr} / \mathrm{CysC}$ ratio is the most predictive of sarcopenia in patients with gastric cancer which is positively correlated with SMI and SMA among the three biomarkers of serum $\mathrm{Cr}$, serum $\mathrm{Cys} C$ and serum $\mathrm{Cr}$ / $\mathrm{Cys} C$ ratio in this study. Previous studies have shown that serum $\mathrm{Cr}$ is related to muscle mass $[35,36]$. In our study, serum $\mathrm{Cr}$ is positively correlated with SMI and SMA which is consistent with the results of previous studies. Serum $\mathrm{Cys} C$ does not show any correlation with muscle mass. As an indicator of renal function, serum CysC has received more and more attention in recent years. It is a low molecular weight protein with a stable production rate and can be freely filtered by the glomerulus [19]. Therefore, the $\mathrm{Cr} / \mathrm{CysC}$ ratio obtained by calibrating $\mathrm{Cr}$ with $\mathrm{CysC}$, that is not affected by muscle mass, can predict sarcopenia.

We found that the best cut-off value of the optimal serum $\mathrm{Cr} / \mathrm{CysC}$ ratio for predicting sarcopenia in patients with gastric cancer is 0.67 . A study found that the optimal cut-off value of $\mathrm{Cr} / \mathrm{CysC}$ ratio in diabetic patients for predicting sarcopenia was 0.9 [11], while another study found that the optimal cut-off value of $\mathrm{Cr} / \mathrm{CysC}$ ratio was 0.71 in COPD patients [30]. However, there is no study to detect the optimal cut-off value of $\mathrm{Cr} / \mathrm{Cys} \mathrm{C}$ ratio for sarcopenia in gastric cancer patients as we know.

The OS of the high serum $\mathrm{Cr} / \mathrm{Cys} \mathrm{C}$ group defined by the optimal cut-off value was significantly higher than that of the low serum $\mathrm{Cr} / \mathrm{CysC}$ group. And sarcopenia shorted the survival time of patients as an independent factor affecting the prognosis of gastric cancer patients which result is consistent with that of $\mathrm{Cr} / \mathrm{Cys} \mathrm{C}$ ratio. We also constructed height-calibrated $\mathrm{Cr} / \mathrm{CysC}$ ratio and SMI and survival models. Studies have shown that 

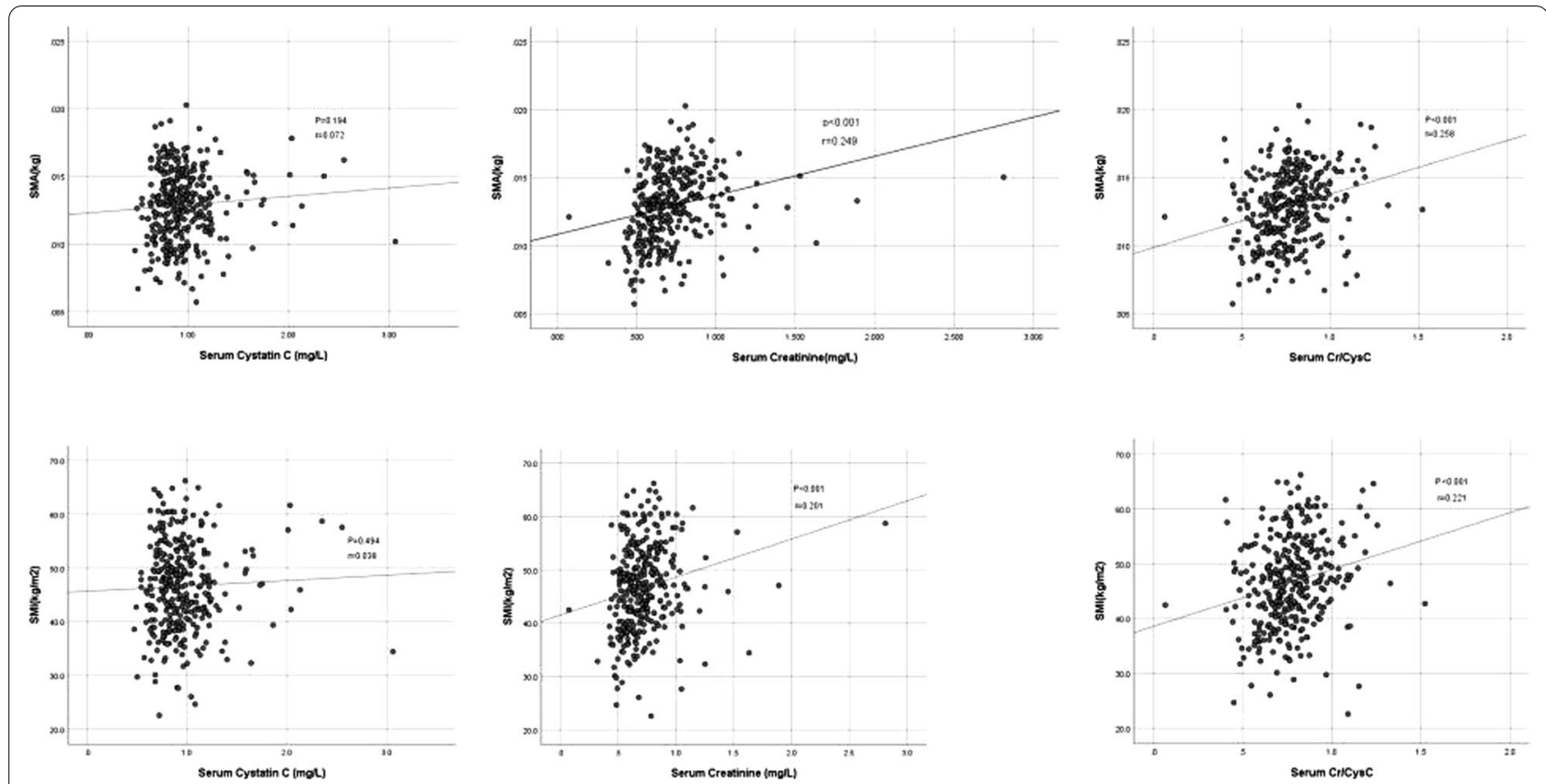

Fig. 2 Linear correlation between the skeletal muscle mass index (SMI), skeletal muscle area (SMA) and cystatin C, creatinine and creatinine/cystatin $C$ ratio in the group

\section{Standard Group}

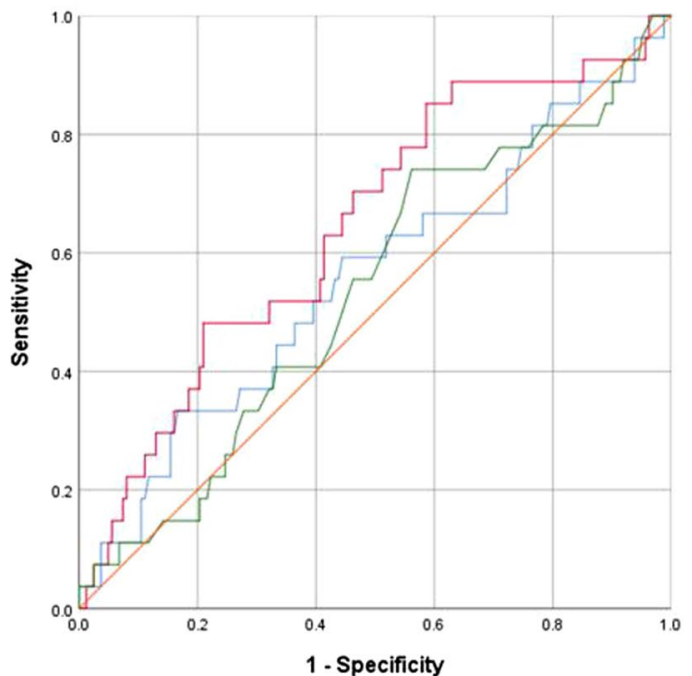

\section{Low skeletal muscle mass Group}

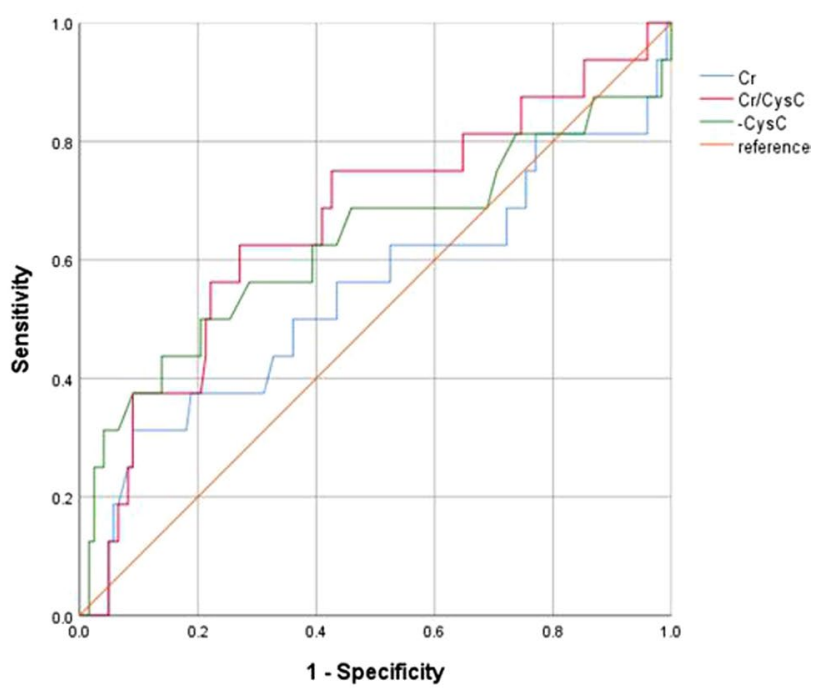

Fig. 3 Receiver operator characteristic curves presenting sarcopenia and low skeletal muscle mass according to serum creatinine (Cr)/cystatin C (CysC) ratio, serum CysC and serum Cr levels

although the $\mathrm{Cr} / \mathrm{Cys} \mathrm{C}$ ratio is related to $\mathrm{OS}$, the correlation is not as good as SMI. But its correlation is greater than SMI when it is used together with SMI as an indicator to measure the prognosis of patients with gastric cancer. This means that the prognosis of gastric cancer patients can be predicted by using the $\mathrm{Cr} / \mathrm{CysC}$ ratio and SMI as a combined index. 

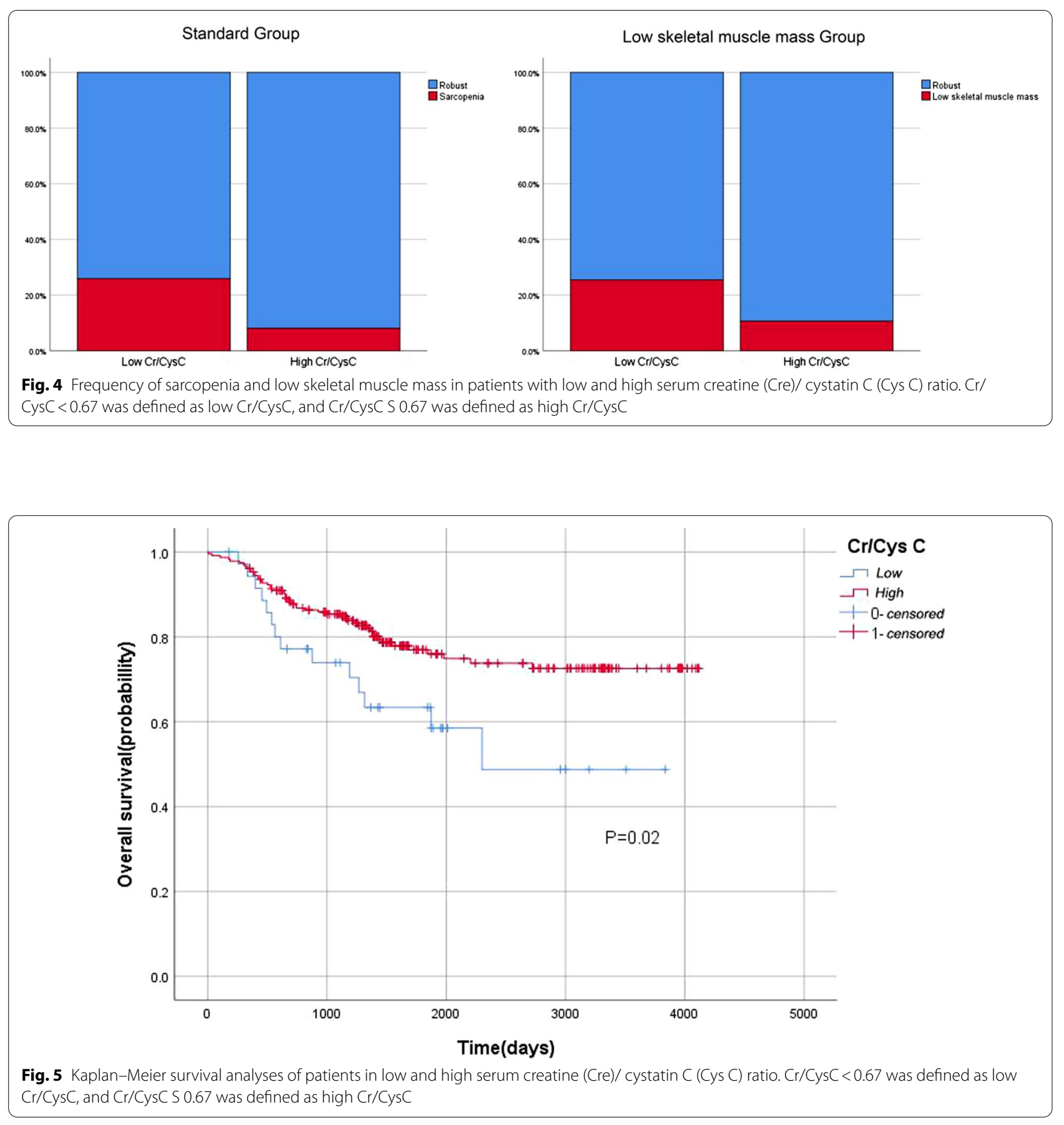

Table 3 Univariate and multivariate analyses of factors in relation to overall survival

\begin{tabular}{lllll}
\hline & \multicolumn{2}{l}{ Univariate analysis } & & \multicolumn{2}{l}{ Multivariate analysis } \\
\cline { 2 - 3 } & HR $(\mathbf{9 5 \%}$ Cl) & p value & HR (95\% Cl) \\
\hline Serum Cr/Cys C low/high & $0.501(0.277-0.906)$ & $0.022^{*}$ & $0.565(0.311-1.025)$ & 0.060 \\
\hline
\end{tabular}

We use age, BMI, pTNM stage and tumor size to perform COX regression to perform survival multivariate analysis Asterisks indicate meaningful results 
In this study, we used the area of all skeletal muscle on the CT scan at the L3 level as the standard for estimating the patient's skeletal muscle mass. Many studies have confirmed that CT scans are an effective way to assess body composition and can predict sarcopenia in the population. It has been widely used in oncology as a highly feasible method. However, it is still subject to many limitations in actual clinical applications as a method of evaluating body composition, such as its high radioactivity and high cost. In addition, the measurement of grip strength and pace is often used to assess sarcopenia. But this method is very dependent on patient compliance and is often difficult to implement in clinical operations. However, early diagnosis and intervention are very important since sarcopenia can significantly affect the prognosis of patients with gastric cancer. Serum $\mathrm{Cr} / \mathrm{CysC}$ ratio is a simple, easy and low-cost method that can initially screen patients for sarcopenia and assist subsequent treatment and intervention. If the $\mathrm{Cr} / \mathrm{Cys} \mathrm{C}$ ratio is lower than 0.67, detailed examinations must be carried out by BIA, DEXA, CT or MRI.

This study also has certain limitations. First of all, it is a retrospective study and sometimes there are certain deficiencies when collecting follow-up information. Second, the sample size is insufficient because this study only included data from a tertiary center for statistical analysis. The detection of serum $\mathrm{Cr}$ and serum $\mathrm{Cys} C$ will also be affected by reagents and instruments. In followup studies, prospective studies can be carried out around this point of view and verified in other centers. Third, this study only referred to the Chinese population. Due to the different criteria for sarcopenia, this limits the applicability of the conclusions, especially for Western populations. In follow-up research, how to determine the appropriate standards according to different groups of people to verify our conclusions is very important.

\begin{abstract}
Abbreviations
Cr/CysC: Serum creatinine/cystatin C; CT: Computed tomography; ASA: American Society of Anesthesiologists; SMI: Skeletal muscle mass index; EWGSOP: European Working Group on Sarcopenia in Older People; AWGS: Asian Working Group for Sarcopenia; OS: Overall survival; IQR: Interquartile range; ROC: Receiver operating characteristic; AUC: Area under the ROC curve; $\mathrm{Cl}$ : Confidence interval; COPD: Chronic obstructive pulmonary disease; ICU: Intensive care unit; MRI: Magnetic resonance imaging.
\end{abstract}

\section{Acknowledgements}

We gratefully acknowledge all staff members of Department of Gastrointestinal Surgery, The Second Affiliated Hospital, Wenzhou Medical University, Wenzhou, Zhejiang, China, for their help and cooperation.

\section{Authors' contributions}

ZS and XS are the main authors of manuscript and have made substantial contributions to the conception and design of study. XY, TC, HY, XW, CZ, TZ, $\mathrm{DC}, \mathrm{ZS}$ and JP have been involved in collection and analysis of the data, ZS and XS gave final approval and revised of the manuscript. All authors read and approved the final manuscript.

Funding

Not applicable.

Availability of data and materials

The data that support the fndings of this study are available from the corresponding author upon reasonable request. Emails could be sent to the address below to obtain the shared data: shenxian@wmu.edu.cn.

\section{Declarations}

\section{Ethics approval and consent to participate}

The research was in compliance with the Declaration of Helsinki. Ethical approval was obtained from the Second Affiliated Hospital, Wenzhou Medical University (ID: 2014063) and the necessity for informed consent was waived.

\section{Consent for publication}

Not applicable.

\section{Competing interests}

The authors declare that they have no competing interests.

\section{Author details}

'Department of Gastrointestinal Surgery, The Second Affiliated Hospital, Wenzhou Medical University, Wenzhou, Zhejiang, China. ${ }^{2}$ Department of Gastrointestinal Surgery, The First Affiliated Hospital, Wenzhou Medical University, Wenzhou, Zhejiang, China.

Received: 11 July 2021 Accepted: 22 December 2021

Published online: 19 January 2022

\section{References}

1. Bray F, Ferlay J, Soerjomataram I, Siegel RL, Torre LA, Jemal A. Global cancer statistics 2018: GLOBOCAN estimates of incidence and mortality worldwide for 36 cancers in 185 countries. CA Cancer J Clin. 2018;68(6):394-424

2. Kim Y, Ejaz A, Spolverato G, et al. Conditional survival after surgical resection of gastric cancer: a multi-institutional analysis of the us gastric cancer collaborative. Ann Surg Oncol. 2015;22(2):57-64.

3. Morley JE, Baumgartner RN, Rouben off R, Mayer J, Nair KS. Sarcopenia. J Lab Clin Med. 2001;137(4):231-43.

4. Zhuang C-L, Shen X, Zou H-B, et al. EWGSOP2 versus EWGSOP1 for sarcopenia to predict prognosis in patients with gastric cancer after radical gastrectomy:analysis from a large-scale prospective study. Clin Nutr. 2019.

5. Drissi M, Cwieluch O, Lechner P, Radziwill R, Vehling-Kaiser U, Hengst K, Masin M. Nutrition care in patients with cancer: a retrospective multicenter analysis of current practice-indications for further studies? Clin Nutr. 2014. https://doi.org/10.1016/j.clnu.2014.03.002.

6. James EJ, et al. Sarcopenia is associated with toxicity in patients undergoing neo-adjuvant chemotherapy for oesophago-gastric cancer. Eur I Surg Oncol. 2015.

7. Viollet L, Gailey S, Thornton DJ, Friedman NR, Flanigan KM, Mahan JD, et al. Utility of cystatin C to monitor renal function in Duchenne muscular dystrophy. Muscle Nerve. 2009;40:438-42.

8. Cartin-Ceba R, Afessa B, Gajic O. Low baseline serum creatinine concentration predicts mortality in critically ill patients independent of body mass index. Crit Care Med. 2007;35:2420-3.

9. Vinge $E$, Lindergård $B$, Nilsson-Ehle $P$, et al. Relationships among serum cystatin C, serum creatinine, lean tissue mass and glomerular filtration rate in healthy adults. Scand J Clin Lab Invest. 1999;59:587-92.

10. Anders G. Cystatin $C$ is indispensable for evaluation of kidney disease. EJIFCC. 2017;28:268-76.

11. Kashani KB, Frazee EN, Kukrálová L, Sarvottam K, Herasevich V, Young PM, et al. Evaluating muscle mass by using markers of kidney function: development of the sarcopenia index. Crit Care Med. 2017;45:e23-9. 
12. Kashani K, Sarvottam K, Pereira NL, Barreto EF, Kennedy CC. The sarcopenia index: a novel measure of muscle mass in lung transplant candidates. Clin Transplant. 2018;32:13182.

13. Osaka T, Hamaguchi M, Hashimoto Y, Ushigome E, Tanaka M, Yamazaki $M$, et al. Decreased the creatinine to cystatin $C$ ratio is a surrogate marker of sarcopenia in patients with type 2 diabetes. Diabetes Res Clin Pract. 2018;139:52-8

14. Suzuki K, Furuse H, Tsuda T, Masaki Y, Okazawa S, Kambara K, et al. Utility of creatinine/cystatin $\mathrm{C}$ ratio as a predictive marker for adverse effects of chemotherapy in lung cancer: a retrospective study. J Int Med Res. 2015;43:573-82

15. Huhmann MB, Cunningham RS. Importance of nutritional screening in treatment of cancer-related weight loss. Lancet Oncol. 2005:6:334-43.

16. Mourtzakis M, Prado CMM, Lieffers JR, Reiman T, McCargar LJ, Baracos VE. A practical and precise approach to quantification of body composition in cancer patients using computed tomography images acquired during routine care. Appl Physiol Nutr Metab. 2008;33(5):997-1006.

17. Prado CMM, Lieffers JR, McCargar $L$, et al. Prevalence and clinical implications of sarcopenic obesity in patients with solid tumours of the respiratory and gastrointestinal tracts: a population-based study. Lancet Oncol. 2008;9(7):629-35.

18. Cruz-Jentoft AJ, Baeyens JP, Bauer JM, et al. Sarcopenia: European consensus on definition and diagnosis: report of the European working group on sarcopenia in older people. Age Ageing. 2010;39(4):412e23.

19. Chen L-K, Woo J, Assantachai P, et al. Asian working group for sarcopenia:2019 consensus update on sarcopenia diagnosis and treatment. J Am Med Dir Assoc. 2020;21(3):300-7.

20. Zhuang C-L, Huang D-D, Pang W-Y, et al. Sarcopenia is an independent predictor of severe postoperative complications and long-term survival after radical gastrectomy for gastric cancer: analysis from a large-scale cohort. Medicine (Baltim). 2016;95(13):e3164.

21. Baumgartner RN, Waters DL. 80. Sarcopenia and sarcopenic-obesity. Principles and practice of geriatric medicine, Volume 2, Fourth Edition.

22. Fukuda Y, Yamamoto K, Hirao M, Nishikawa K, Nagatsuma Y, Nakayama T, Tanikawa S, Maeda S, Uemura M, Miyake M, Hama N, Miyamoto A, Ikeda M, Nakamori S, Sekimoto M, Fujitani K, Tsujinaka T. Sarcopenia is associated with severe postoperative complications in elderly gastric cancer patients undergoing gastrectomy. Gastric Cancer. 2016;19(3):986-93. https://doi.org/10.1007/s10120-015-0546-4.

23. Kawamura T, Makuuchi R, Tokunaga M, Tanizawa Y, Bando E, Yasui H, Aoyama T, Inano T, Terashima M. Long-term outcomes of gastric cancer patients with preoperative sarcopenia. Ann Surg Oncol. 2018;25(6):162532. https://doi.org/10.1245/s10434-018-6452-3.

24. Kudou K, Saeki H, Nakashima Y, Edahiro K, Korehisa S, Taniguchi D, Tsutsumi R, Nishimura S, Nakaji Y, Akiyama S, Tajiri H, Nakanishi R, Kurashige J, Sugiyama M, Oki E, Maehara Y. Prognostic significance of sarcopenia in patients with esophagogastric junction cancer or upper gastric cancer. Ann Surg Oncol. 2017;24(7):1804-10. https://doi.org/10.1245/ s10434-017-5811-9.

25. Shen $Y$, Hao Q, Zhou J, Dong B. The impact of frailty and sarcopenia on postoperative outcomes in older patients undergoing gastrectomy surgery: a systematic review and meta- analysis. BMC Geriatr. 2017;17(1):188. https://doi.org/10.1186/s12877-017-0569-2.

26. Maconi G, Manes G, Porro GB. Role of symptoms in diagnosis and outcome of gastric cancer. World J Gastroenterol. 2008;14(8):1149-55.

27. Correia MI, Waitzberg DL. The impact of malnutrition on morbidity, mortality, length of hospital stay and costs evaluated through a multivariate model analysis. Clin Nutr. 2003;22(3):235-9.

28. Choi WJ, Kim J. Nutritional care of gastric cancer patients with clinical outcomes and complications: a review. Clin Nutr Res. 2016;5(2):65-78.

29. Syuichi T, Mitsuya M, Kunihiko I, Imaharu N. Creatinine/cystatin C ratio as a surrogate marker of residual muscle mass in amyotrophic lateral sclerosis. Neurol Clin Neurosci. 2013;1:32-7.

30. Kusunoki H, Tsuji S, Wada Y, Fukai M, Nagai K, Itoh M, et al. Relationship between sarcopenia and the serum creatinine/cystatin C ratio in Japanese rural community-dwelling older adults. JCSM Clin Rep. 2018;3:e00057.

31. Barreto EF, Poyant JO, Coville HH, Dierkhising RA, Kennedy CC, Gajic O, et al. Validation of the sarcopenia index to assess muscle mass in the critically ill: a novel application of kidney function markers. Clin Nutr. 2019:38:1362-7
32. Inker LA, Astor BC, Fox CH, Isakova T, Lash JP, Peralta CA, et al. KDOQI US commentary on the $2012 \mathrm{KDIGO}$ clinical practice guideline for the evaluation and management of CKD. Am J Kidney Dis. 2014;63:713-35.

33. Isoyama N, Qureshi AR, Avesani CM, Lindholm B, Bàràny P, Heimbürger $\mathrm{O}$, et al. Comparative associations of muscle mass and muscle strength with mortality in dialysis patients. Clin J Am Soc Nephrol CJASN. 2014;9:1720-8.

34. Yang J, Zhang T, Feng D, Dai X, LvT, Wang X, Gong J, Zhu W, Li J. A new diagnostic index for sarcopenia and its association with short-term postoperative complications in patients undergoing surgery for colorectal cancer. Colorectal Dis. 2019;21(5):538-47. https://doi.org/10.1111/codi. 14558.

35. Thongprayoon C, Cheungpasitporn W, Kashani K. Serum creatinine level, a surrogate of muscle mass, predicts mortality in critically ill patients. J Thorac Dis. 2016;8:E305e11.

36. Udy AA, Scheinkestel C, Pilcher D, Bailey M. Australian and New Zealand Intensive Care Society Centre for Outcomes and Resource Evaluation. The association between low admission peak plasma creatinine concentration and in-hospital mortality in patients admitted to intensive care in Australia and New Zealand. Crit Care Med. 2016;44:73-82.

\section{Publisher's Note}

Springer Nature remains neutral with regard to jurisdictional claims in published maps and institutional affiliations.

Ready to submit your research? Choose BMC and benefit from

- fast, convenient online submission

- thorough peer review by experienced researchers in your field

- rapid publication on acceptance

- support for research data, including large and complex data types

- gold Open Access which fosters wider collaboration and increased citations

- maximum visibility for your research: over $100 \mathrm{M}$ website views per year

At BMC, research is always in progress.

Learn more biomedcentral.com/submissions 